\title{
LA EUROPEIZACIÓN DE LA REPÚBLICA DE MOLDOVA: UN CAMINO SINUOSO E INCIERTO HACIA LA INTEGRACIÓN EUROPEA
}

\author{
Aurelia Dercaci ${ }^{1}$ \\ Universidad de Deusto
}

\begin{abstract}
Resumen:
Después de la proclamación de la independencia en 1991, la República de Moldova (RM) ha declarado su firme determinación de emprender su camino hacia Europa, a la que se siente identificada culturalmente. Aunque en las declaraciones y documentos adoptados el Estado nunca ha renunciado a este objetivo, ciertas acciones de sus líderes políticos parecen contradecir dicho compromiso. El trabajo tiene como objetivo analizar el proceso de evolución de la República de Moldova a la Unión Europea (UE), los compromisos asumidos por ambos y su cumplimiento. Además, voy a comparar los progresos realizados por Ucrania y RM hacia el acercamiento a la Unión Europea en los últimos años. Hace algunos años, Ucrania fue el país oriental con la perspectiva fundamental de la futura integración en la UE debido a sus cambios democráticos, hoy República de Moldova ha demostrado ser capaz de hacer más progresos en la promoción de la democracia y la aplicación de las reformas de gobernanza que Ucrania. Por otro lado, la relación entre la RM y la UE sigue siendo influenciada por las medidas adoptadas por la Federación de Rusia en la región donde todavía tiene influencia significativa, y tratar de mantener su presencia. Una de las herramientas fuertes de la influencia es la dependencia del suministro de energía y su falta de diversificación. El conflicto sin resolver de Transnistria es otro factor determinante de la falta de certeza en el proceso de europeización de la RM. El trabajo tiene como objetivo analizar la eficiencia de la europeización de la República de Moldova a través de la Política de Vecindad Europea, utilizando un método combinado constructivista y racionalista.
\end{abstract}

Palabras clave: Unión Europea, Política Europea de Vecindad, europeización, República de Moldova, Ucrania.

Title in English: "The Europeanization of the Republic of Moldova: A Winding and Insecure Path to European Integration"

Abstract:

After the proclamation of Independence in 1991, the Republic of Moldova (RM) has declared its strong determination to make its way towards Europe, to which RM feels identified culturally. Although in the statements and adopted documents the state has never given up to this goal, certain actions of its political leaders seem to contradict this commitment. The paper aims to analyze the evolution process of the Republic of Moldova to the European Union (EU), the commitments assumed by both of them and their fulfillment. In addition, I will compare the progress made by Ukraine and RM towards the rapprochement to the European Union in the recent years. Some years ago Ukraine was the Eastern country with the major perspective of future integration in EU due to its democratic changes, nowadays Republic of Moldova has shown to be able to make more progress in promoting democracy and implementing governance reforms than Ukraine. On the other hand, the relationship between RM and EU continues to be influenced by the actions taken by the Russian Federation in the region where it still has significant influence and tries to maintain its presence. One of the strong tools of the influence is the dependence on energy supply and its lack of diversification. The unsettled Transdnestrian conflict is another factor determining the lack of certainty in the process of Europeanization of the RM. The paper aims to analyze the efficiency of the Europeanization of Republic of Moldova through the European Neighborhood Policy using a combined constructivist and rationalist approach.

Keywords: European Union, European Neighbourhood Policy, Europeanization, Republic of Moldova, Ukraine.

Copyright @ UNISCI, 2014.

Las opiniones expresadas en estos artículos son propias de sus autores, y no reflejan necesariamente la opinión de UNISCI. The views expressed in these articles are those of the authors, and do not necessarily reflect the views of UNISCI.

\footnotetext{
1 Aurelia Dercaci es becaria FPI y Doctoranda en el Equipo de Investigación Integración Europea de la Universidad de Deusto. Sus líneas de investigación son: Integración Europea; la UE como poder normativo y actor de gobernanza política y económica; la UE como actor internacional en un mundo multipolar.

E-mail: aurelia.dercaci@deusto.es.
} 


\section{Introducción}

Desde el momento de la desmembración de la ex URSS, la República de Moldova ${ }^{2}$, al igual que las demás repúblicas ex soviéticas, declaró su independencia y procedió a seguir por el difícil camino de la constitución de un Estado independiente, con sus propias instituciones, políticas públicas y política. A la hora de establecer sus relaciones con el resto de mundo, la República de Moldova ha optado por declarar su orientación hacia el Oeste, para formar alguna vez parte de la Unión Europea, pero al mismo tiempo no ha dejado de tener estrechas relaciones con el Este, llegando a formar parte de la Comunidad de Estados Independientes, creada como una organización orientada a ser un substituto de la URSS, esta vez con la participación benévola de todas las exrepúblicas soviéticas.

El objetivo del presente artículo es analizar el paradigma de actuación de Moldova en el ámbito de la política exterior, la evolución de las relaciones entre Moldova y la Unión Europea, determinadas transformaciones o impactos que la UE ha podido tener en las políticas e instituciones de Moldova. El país, aunque no ha dejado en ningún momento de mantener, mediante el discurso y también (aunque en menor medida) mediante acciones políticas, su orientación y vocación europea, no obstante tampoco se ha alejado de la zona de influencia del Este, esto último en parte por razones de pasado común histórico (desde 1939 hasta 1989), y en parte por la interdependencia económica, energética, etc. Nos planteamos ver cuáles han sido los puntos cruciales de las relaciones de Moldova con la UE, en qué momentos las actuaciones de las dos partes se han demostrado más eficientes y cuando en cambio ha habido momentos críticos.

Explicaremos brevemente el problema del conflicto transnistrense que está considerado como el obstáculo principal para cualquier perspectiva, aunque lejana, para Moldova de llegar a ser candidato a la adhesión. La existencia de este conflicto congelado para más de 20 años determina también en este aspecto una dependencia de la Federación Rusa, que a la vez ha contribuido directa e indirectamente al desencadenamiento del conflicto en cuestión, y que sigue manteniendo en la actualidad fuerzas militares en esta región.

Veremos en perspectiva diacrónica las opciones de política exterior de la República de Moldova después de su independencia, e intentaremos explicar las razones que han determinado los giros y cambios que ha habido.

Nos planteamos aplicar un enfoque teórico combinado del racionalismo y constructivismo para analizar la actuación de los actores implicados: las instituciones de la UE y de los países parte de la Política Europea de Vecindad. En trabajos recientes que analizan la presencia y actuación europea en los países de la Europa Central y del Este y la PEV se señala que las políticas de las dos partes pueden ser analizadas tanto desde la perspectiva racionalista, cuando al tomar una decisión se da prioridad a razones de costebeneficio, como desde la perspectiva constructivista que plantea que los actores están guiados en la toma de decisiones por valores y sentido de la identidad ${ }^{3}$. En el enfoque combinado las

\footnotetext{
${ }^{2}$ La denominación oficial del país al que nos referimos, adoptada en su Declaración de Independencia en 1991 es "Republica Moldova" (República de Moldova). Aunque en castellano se puede encontrar también la denominación "Moldavia", su uso puede llevar a confusiones con la denominación de la antigua región histórica, el Principado de Moldova ("Moldavia" en inglés), o con la modalidad en la que se denominaba la ex República Soviética Socialista Moldava durante la ex URSS.

3 Schimmelfennig, Frank, (2003): The EU, NATO and the Integration of Europe. Rules and Rhetoric, Cambridge, Cambridge University Press, p.11.
} 
dos teorías se ven más bien como métodos o instrumentos de análisis del proceso, y se complementan mutuamente para una mejor comprensión y reflexión ${ }^{4}$.

\section{Principal instrumento de europeización fuera de las fronteras - la Política Europea de Vecindad (PEV)}

La Comisión Europea formuló por primera vez la Política Europea de Vecindad (PEV) en su Comunicación de marzo de 2003 "Una Europa más amplia" en la que manifestaba la determinación de la UE:

“(...) de evitar nuevas líneas divisorias en Europa y de promover la estabilidad y la prosperidad dentro de sus nuevas fronteras y más allá de ellas (...), la ampliación fortalecerá las relaciones con Rusia".

Asimismo se subrayaba la importancia de que "se estrecharan las relaciones con Ucrania, Moldavia, Bielorrusia y los países del Sur del Mediterráneo sobre la base de un planteamiento a largo plazo de promoción de reformas, del desarrollo sostenible y del comercio",5.

Un posterior y desarrollado Documento Estratégico sobre la $\mathrm{PEV}^{6}$ le siguió en mayo de 2004, aportando el marco concreto para una Política de Vecindad y la información sobre cómo debería funcionar.

En 2006 la Comisión Europea hizo un análisis de la evolución de la PEV y en una Comunicación al Consejo y al Parlamento subrayó los puntos positivos y las deficiencias de la PEV hasta el momento respectivo. Entre los primeros se destacan el aspecto de la integración, mediante el cual se proporciona un marco único y claro que abarca el ámbito de la vecindad en su conjunto sin reducirse exclusivamente a las cuestiones económicas, y extendiéndose también a temas referentes a la gobernanza y derechos humanos. Además, los Planes de Acción, como instrumentos operativos de la Política de Vecindad se adoptan de común acuerdo por las partes implicadas, por lo cual no se trata de imposición alguna. Como otros puntos positivos de la evolución hasta el momento respectivo se señalan el carácter concreto y detallado de los Planes de Acción, y el mejor empleo de los fondos, ya que a través del nuevo instrumento financiero IEVA se planificaba conceder la asistencia comunitaria con mayor eficacia, siendo determinada por las políticas a promover, y aprovechando las nuevas formas de cooperación: la transfronteriza, el TAIEX (Technical Assistance and Information Exchange Instrument), el hermanamiento, etc., disponiendo de mayores recursos.

Entre los aspectos que se plantea mejorar, la Comunicación de la Comisión indica la integración comercial y económica, dado que aunque se había continuado estrechando las relaciones comerciales con la mayoría de los socios de la PEV, (en concreto se ha respaldado el proceso de adhesión de Ucrania a la OMC, se había preparado la negociación de un acuerdo

\footnotetext{
${ }^{4}$ Kratochvil, Petr, y Tulmets Elsa (2010): Constructivism and rationalism in EU External Relations. The case of the ENP, Baden-Baden, Nomos.

${ }^{5}$ Comunicación de la Comisión Europea COM (2003) 104 final: "Una Europa más amplia. Relaciones con los países vecinos: un nuevo marco para las relaciones con nuestros vecinos del Este y del Sur de Europa", en http://eur-lex.europa.eu/LexUriServ/LexUriServ.do?uri=COM:2003:0104:FIN:ES:PDF

${ }^{6}$ Comunicación de la Comisión Europea COM (2004) 373 final, "European Neighbourhood Policy, Strategy Paper", en http://ec.europa.eu/world/enp/pdf/strategy/strategy_paper_en.pdf.
} 
de libre comercio y concesión de preferencias comerciales autónomas a Moldova, y negociado varios acuerdos amplios de cooperación en el dominio de los servicios y agricultura con los socios mediterráneos), no obstante se subraya la importancia de ofrecer a todos los miembros de la PEV una clara perspectiva de integración comercial y económica con Europa para que todas las partes puedan obtener mayores ventajas económicas y políticas. Se señala además como un área en la que se debe seguir trabajando la movilidad y migración, ya que pese a algunos pasos dados en esta dirección, "la lentitud y el coste de los trámites de expedición de visados de corta duración tienen un efecto disuasorio muy notorio ante los países socios"7.

Otro tema de mayor importancia es el referente a los conflictos regionales, en relación a los cuales señala:

\begin{abstract}
“A pesar de algunos logros concretos (en relación con la gestión de las fronteras (...)), la PEV apenas si ha contribuido a la resolución de los conflictos en la región, ya sean abiertos o enquistados. La Unión Europea ha de ser más activa y estar más presente en los mecanismos regionales y multilaterales de resolución de conflictos y en las iniciativas de observación o mantenimiento de la paz."
\end{abstract}

La Comunicación respectiva, de importancia estratégica, concluye subrayando la necesidad de que la UE asuma los costes y haga esfuerzos en vista de lograr los objetivos establecidos, pero también señala la importancia de la implicación de los países beneficiarios en la consecución de los compromisos asumidos al respecto. En estas mismas conclusiones, la Comisión planifica una reunión en la que las partes de la PEV, la Comisión por un lado y los países vecinos por otro, compartan sus opiniones con respecto a la evolución y las mejoras de la PEV.

La siguiente Comunicación estratégica de la Comisión se publica en 2007 y se titula “A Strong European Neighbourhood Policy"9. En ésta se hace hincapié en unos puntos muy importantes para la evolución de la PEV y que son la diferenciación, la apropiación y los procesos regionales. Dado que la PEV incluye países muy diferentes en una única política, resulta de suma importancia que el tratamiento de las relaciones con cada uno de estos sea flexible y diferenciado en función de la situación política de cada socio, de su nivel de ambiciones en la promoción de las reformas y en su aspiración hacia la UE y su nivel de desarrollo socio-económico.

Aunque la UE ha reconocido el criterio de la diferenciación, y los acuerdos y negociaciones existentes lo demuestran, no obstante cabe destacar la insatisfacción de Ucrania, por ejemplo, y de Moldova, en este sentido, que consideran que debido a su vocación y aspiración europea, merecen que la UE les ofrezca perspectivas de adhesión en condiciones más favorables, entendiendo así la diferenciación.

En los años siguientes la PEV ha sufrido toda una serie de re-denominaciones y transformaciones: "Wider Europe", "ENP", "ENP+", "BlackSea Energy", "Enhanced ENP" y

\footnotetext{
${ }^{7}$ Comunicación de la Comisión Europea COM (2006) 726, “On Strengthening The European Neighbourhood Policy" p. 4, en http://eur-lex.europa.eu/LexUriServ/LexUriServ.do?uri=COM:2006:0726:FIN:EN:PDF.

${ }^{8}$ Comunicación de la Comisión Europea COM (2006) 726, op.cit., p.4.

${ }^{9}$ Comunicación de la Comisión Europea COM (2007) 774 final: “A Strong European Neighbourhood Policy", p. 2 en http://ec.europa.eu/world/enp/pdf/com07 774_en.pdf.
} 
en 2009 "Eastern Partnership" (que es una parte de la política respectiva referente solo a los países del Este), lo que reflejaba la insatisfacción de las instituciones de la UE con la evolución de la política y con la eficiencia en la consecución de los objetivos que esta se planteaba. Por otra parte tampoco los países vecinos han estado contentos con lo que la política contemplaba y ofrecía. En el caso de Moldova y Ucrania, que siempre han aspirado a la integración europea, incluyendo este objetivo en sus planes estratégicos, la PEV les proponía comprometerse con las reformas, ofrecerles determinada asistencia orientada a realizarlas, pero excluía por completo la perspectiva de adhesión.

En 2012 una nueva Comunicación conjunta de la Alta Representante para Asuntos Exteriores de la $\mathrm{UE}^{10}$ manifiesta la importancia de replantear la relación con los países vecinos, indicando como una de las nuevas características de la misma el principio de "dar más para recibir más" y el de la responsabilidad mutua, así como la diferenciación, subrayando a la vez la importancia de la seguridad, estabilidad y democracia, especialmente después de las revoluciones de los países árabes.

La PEV puede ser considerada como una europeización de estados no miembros, porque los impulsa a realizar reformas y adoptar determinadas políticas, al igual que a los estados miembros o candidatos, pero con la diferencia de que a los estados vecinos a los que está dirigida no se les ofrece la perspectiva de adhesión. En este contexto, la europeización se manifiesta como una inclusión política que presupone la transferencia de la legislación y formas de gobernanza, inclusión de mercado a través de la expansión del mercado común y asistencia financiera, a la que le sigue la inclusión normativa, referida a la puesta en aplicación de normas y efectos de socialización y la inclusión identitaria - sentimientos de pertenencia e identidad $^{11}$. Otros autores señalan que la PEV puede ser vista como el más parecido caso de europeización más allá de las fronteras de Europa, porque se dirige a vecinos cercanos, abarca una serie de políticas y se basa en el compromiso explícito de la UE de extender el acervo comunitario más allá de los países miembros ${ }^{12}$ o por otra parte como una "ampliación-light" ${ }^{13}$ o bien como "europeización de la vecindad"14.

Los principales mecanismos de europeización de estados no miembros son la condicionalidad, cuando la UE ofrece a los gobiernos de los países asistencia, ayuda, recursos financieros, accesos a mercados, mayor cooperación a cambio de que estos realicen las transformaciones recomendadas; y la socialización, proceso en el que los países promueven las reformas y cambios en sus políticas porque conocen y aprenden mediante conexiones sociales el modelo de la UE, y lo adoptan por considerarlo apropiado y legitimado.

En el caso de la PEV la condicionalidad es el principal mecanismo aplicado, pero también la transferencia de normas se puede llevar a cabo cuando hay una condicionalidad

\footnotetext{
${ }^{10}$ Comunicación de la CE, JOIN (2012) 14 final: "Diseño de una nueva Política Europea de Vecindad".

11 Schmitke, Oliver y Chira-Pascanut, Constantin ,"The promise of Europe: Moldova and the Process of Europeanization", en Schmidtke, Oliver, yYekelchyk, Serhy, (eds.) (2008): Europe's Last Frontier? Belarus, Moldova and Ukraine between Russia and the European Union, Palgrave Macmillan, p. 146-151.

${ }^{12}$ Schimmelfennig, Frank: "Europeanization beyond the member States", en http://www.eup.ethz.ch/people/schimmelfennig/publications/10_ZSE_Europeanization_manuscript_pdf

${ }^{13}$ Popescu, Nicu y Wilson, Andrew (2009): The limits of enlargement- lite: European and Russian power in the troubled neighbourhood, London, ECFR.

${ }^{14}$ Gawrich, Andrea, Melnykovska, Inna and Schweickert, Rainer: "Neighbourhood Europeanization through ENP: The Case of Ukraine”, Journal of Common Market Studies, vol. 48, Issue 5 (November 2010), pp. 12091235.
} 
frágil pero el país considera adecuadas las reformas o las políticas recomendadas en el marco de los Planes de Acción.

\section{PEV en Moldova}

Las relaciones de Moldova con la UE han empezado en 1994, y se han basado en un Acuerdo de Partenariado y Colaboración (APC) que entró en vigor en julio de 1998, aunque durante varios años este no se hizo efectivo.

En 2005 en el marco de la PEV fue firmado el Plan de Acciones Unión Europea República de Moldova (PAUERM) para un período de tres años. Aunque el plazo de vigencia del Plan venció, este sigue siendo relevante, ya que hasta 2013 la asistencia dirigida al país por parte de la UE se hará en base a dicho documento. El 12 de enero de 2010 la República de Moldova y la UE acordaron la negociación de un Acuerdo de Asociación que substituirá el APC, y en paralelo se ha elaborado por los representantes de la UE y el Gobierno de Moldova el Plan de Acciones en el dominio de la liberalización del régimen de visados.

En 2011 la Comisión Europea empezó el proceso de reforma de la PEV como resultado de las revoluciones en la vecindad Sur de la UE y también en respuesta al regreso democrático en los estados de la vecindad del Este. La PEV revisada enfatiza el principio de diferenciación que contempla que el nivel de profundización de las relaciones entre la UE y los estados partners, así como el volumen de asistencia ofrecida por la UE, están determinados por los avances en la promoción de las reformas. Los criterios en base a los cuales la UE evaluará el éxito de los estados se referirán en primer lugar en la promoción y consolidación de los valores democráticos, estado de derecho, respeto de los derechos humanos y libertades fundamentales, la independencia de la justicia, la lucha contra la corrupción, la libertad de expresión de los medios de comunicación y la consolidación de la sociedad civil.

En el último informe de progreso sobre la implementación de la PEV en Moldova ${ }^{15}$ se subrayan los importantes avances que el país ha registrado. Se menciona que el país ha hecho considerables progresos en la implementación de la primera fase del Plan de Acciones sobre la liberalización de visados y se han dado pasos importantes para la resolución pacífica del conflicto transnistrense. Se ha mantenido la estabilidad macroeconómica y se ha registrado un crecimiento sostenido aunque los indicadores económicos han bajado a causa del entorno externo difícil. La mejora del clima de inversiones y la realización de reformas estructurales en educación y en el sector energético son otros aspectos positivos señalados en el informe. De hecho tras la adhesión en 2010 a la Comunidad energética Europea, en 2011 Moldova ha ejercido con éxito la presidencia rotatoria de la Comunidad y ha registrado avances en el cumplimiento de sus obligaciones en la misma.

En el informe se hacen también una serie de recomendaciones que el país ha de seguir, y que se refieren a la necesidad de implementar estrategias de reformas del sector de la justicia para la protección de los derechos humanos y para la lucha contra la corrupción, de hacer reformas en la administración pública para consolidar la transparencia, iniciar un

\footnotetext{
${ }^{15}$ European Commission, High Representative of the European Union For Foreign Affairs And Security Policy, SWD (2013) 80 final: "Implementation of the European Neighbourhood Policy in Republic of Moldova. Progress in 2012 and recommendations for action".
} 
dialogo más activo y pragmático para buscar medidas de resolución del conflicto transnistrense, continuar las reformas a largo plazo en el dominio de la educación y la sanidad.

\subsection{El camino sinuoso de Moldova}

Tras la declaración de su independencia en 1991 la República de Moldova ha empezado a construir sus políticas e instituciones como estado independiente, trazando sus estrategias y perspectivas ${ }^{16}$. La dirección que el país veía en aquel momento como más idónea y prioritaria era la vía europea. A partir de 1990 este empezó a ser el discurso político principal, y a partir de 1994 se materializó en la firma de un documento que consagraba la orientación europea de Moldova y se asumía el compromiso de acometer transformaciones para alcanzar la meta. Aun así los siguientes aproximadamente 15 años el camino de Moldova ha distado mucho de ser recto, y sólo hace unos pocos años parece haberse reducido (pero no desaparecido por completo) el riesgo de descarrilarse del rumbo europeo. Korosteleva (2012) distingue tres etapas en la relación de Moldova con la Unión Europea: 1991 - 1994, cuando el país estaba en busca de su identidad, 1994 - 2009, en el que se ha avanzado en la dirección europea, pero por un camino ondeante, y desde 2010 hasta la fecha cuando se emprenden acciones estratégicas orientadas a la integración europea. ${ }^{17}$

Desde 2010, después de la llegada al poder de la Alianza para la Integración Europea constituida por cuatro partidos de orientación democrática, las actuaciones orientadas al acercamiento a la Unión Europea y ahora basadas en transformaciones reales han sido cada vez más frecuentes y el compromiso europeo mayor ${ }^{18}$. Se han intentado resolver importantes problemas referentes a la consolidación de las capacidades administrativas para la puesta en marcha de reformas estructurales.

Este período ha sido marcado también por la importante ayuda financiera orientada a la promoción de las reformas y apoyo de la economía moldava que ha sido concedida por el FMI, el Banco Mundial y por la UE.

Como hemos mencionado, el diálogo con la UE se lleva a cabo mediante el Plan de Acciones $^{19}$ que fue firmado en 2005 para 3 años pero que se prorrogó en 2008. Al mismo tiempo siguieron su curso las negociaciones para la firma del Acuerdo de Asociación, que fue rubricado en noviembre de 2013.

Moldova ha intentado demostrar en estos años mediante hechos que está decidida a llevar a cabo importantes y complejas reformas. La idea de un período de estabilidad que se esperaba tras la llegada al poder de la Alianza para la Integración Europea ha sido bien acogida en la UE. La Unión contemplaba aplicar el principio de "más por más": a países con mayor nivel de compromiso y mayores éxitos en las reformas económicas y políticas, se les iba a conceder más ayuda para incentivar su realización.

\footnotetext{
16 "Declaration of Independence of the Republic of Moldova", en http://www.presedinte.md/eng/declaration.

${ }^{17}$ Korosteleva, Elena: “EU partnership/governance in Moldova. An unrequited “partner?”, in Korosteleva, Elena (2012): The European Union and its Eastern Neighbours. Towards a more ambitious partnership?, Routledge, New York, p. 105.

18 "Evoluciones en las relaciones de la República de Moldova con la UE", Documento del Ministerio de Asuntos Externos e Integración Europea, en http://www.mfa.gov.md/din-istoria-rm-ue/.

${ }^{19}$ European Union - Moldova Action Plan, en

http://ec.europa.eu/world/enp/pdf/action_plans/moldova_enp_ap_final_en.pdf.
} 
Moldova parece encaminada firmemente hacia la Unión Europea, pero siempre se deben tener en cuenta los posibles riesgos y presiones externas o internas que podrían contribuir a otro cambio de rumbo. Aunque Moldova vea en la aproximación a Europa el motor del cambio y así lo proclame, la situación sigue siendo impredecible.

La escasa estabilidad en la orientación de la política externa de Moldova tiene varias explicaciones en nuestra opinión. Entre las primeras estaría la multipolaridad ${ }^{20}$ del mundo actual, ofreciendo a países más pequeños múltiples opciones de asociación y alianzas. El alto coste de las reformas en algunos casos puede ser también un desincentivo a la hora de adoptarlas, aunque esto suponga un retroceso en el proceso de acercamiento a la UE. Por otra parte la existencia de una sociedad y una clase política sin mayor tradición democrática conlleva también un riesgo en este sentido: gobernantes sin mayor tradición política, y con relativamente poca experiencia democrática, la prevalencia de los intereses particulares de estos, el alto nivel de corrupción en las instituciones estatales. Otras razones serían la dependencia económica de Rusia y las políticas que este país dirige hacia su "vecindad cercana".

Algunas actuaciones de las élites políticas de Moldova (y también de Ucrania) en determinados momentos pueden ser explicadas por lo que algunos autores llaman multivectorialismo $^{21}$ y otros neo-titoismo $^{22}$.

Por otra parte varios autores coinciden en que tanto Moldova como Ucrania, que han mantenido siempre la retórica proeuropea y han manifestado la proximidad identitaria de sus países con la UE, durante el período de las relaciones con la UE han tenido mayores expectativas - no sólo y no tanto en términos de apoyo material, sino sobre todo en el ofrecimiento de una perspectiva de mayor acercamiento a la UE y en un futuro la de adhesión a la Unión ${ }^{23}$. Como las expectativas no se han visto cumplidas, los países han experimentado cierto desengaño que también puede contribuir al zigzagueo en el trayecto de los países mencionados.

Moldova parecía en los dos últimos años haberse encaminado con firmeza por el camino de las reformas democráticas y acercamiento a la UE, pero a principios de 2013 ha

\footnotetext{
20 Popescu, Nicu y Wilson, Andrew (2011): Turning presence into power: Lessons from the Eastern Neighbourhood, ECFR.

${ }^{21}$ Gnedina, Elena y Sleptova Evghenia: "The echewing Ucranian politics", CEPS Working Papers, N. 360 (January 2013), en http://www.ceps.eu/book/eschewing-choice-ukraine\%E2\%80\%99s-strategy-russia-and-eu: "Ukraine has long been castigated for its noncommittal attitude to cooperation with the EU, this being part of its 'multi-vector' foreign policy. Such a policy was widely attributed to the failings of domestic élites, which delay reform for fear of losing rents and power. The recent setback in EU-Ukraine relations, however, highlights more complex reasons behind this. First, a pro-European vector is not a self-evident choice for Ukraine, which is economically interdependent with both Russia and the EU. Second, the economic crisis has made the EU less attractive in the short term. In good times business was looking to Europe for opportunities to develop. But in times of crisis it is looking to Russia for cheap resources to survive. Despite these unfavorable short-term trends, an association agreement with the EU stands out as the only alternative that promises to put the shaky Ukrainian economy back on track towards long-term sustainable economic growth".

${ }^{22}$ Popescu, Nicu y Wilson, Andrew (2011): Turning presence into power: Lessons from the Eastern Neighbourhood, ECFR, p. 5.

${ }^{23}$ Raik, Kristi (2012): Moldova deserves more from the EU, FIIA Notes; Parkes, Roderick y Viilup, Elina: "¿Asociación oriental? Algo más parecido a una dependencia mutua. El caso de la Unión Europea y la cooperación en asuntos interiores con Moldavia", CIDOB International Notes (2012); Popescu y Wilson (2011): "Turning presence into power...", op. cit.
} 
demostrado de nuevo que la región es propensa a las crisis ${ }^{24}$. Después de la anterior crisis política en la que durante tres años entre los partidos de la Alianza de gobierno no hubo acuerdo para la elección del Presidente del país ${ }^{25}$, ahora como resultado de la confluencia de algunos de los factores ya mencionados - el nivel reducido de cultura política y la prevalencia de los intereses particulares de determinados políticos en el poder - han causado la dimisión del Gobierno situando al país ante la perspectiva de elecciones anticipadas a falta de 2 años para el fin del mandato. En este mismo período están en curso las negociaciones para la firma del Acuerdo de Asociación, y se contemplaba que en la cumbre de la Asociación Oriental (EaP) del próximo mes de septiembre se iba a poder rubricar el documento para ser firmado en 2014. Los partidos políticos que constituían la Alianza de gobierno estuvieron negociando una posible nueva unión o alianza de gobierno, ya que en aquellos momentos unas elecciones parlamentarias anticipadas podían llevar de nuevo a cambios en la orientación política y de prioridades de relaciones externas de la República de Moldova. La situación de inestabilidad que existía en el país preocupaba a la sociedad y también a la UE, que hizo un llamamiento a la unión entre todas las fuerzas políticas del país para seguir con las reformas empezadas y por la vía de la democracia y el estado de derecho. ${ }^{26}$

Dos meses más tarde, en mayo de 2013, después de duras negociaciones internas, se consiguió elegir al frente del Ejecutivo a Iurie Leanca, ex ministro de exteriores, apoyado por la mayoría de las fuerzas políticas. Moldova logró así mantener la estabilidad política en el país, evitando elecciones anticipadas y manteniendo en el poder la alianza de partidos proeuropeos y por consiguiente la orientación europea del país, confirmada por el primer ministro en su discurso de presentación del nuevo gobierno. ${ }^{27}$

Una vez superada la crisis política en mayo de 2013, el proceso de transformación y acercamiento de Moldova a la UE, que incluía las negociaciones referentes al Acuerdo de Asociación y al Acuerdo de Comercio Libre y Comprehensivo siguió según la agenda planificada, y el pasado 12 de junio se finalizaron las negociaciones sobre las previsiones del mismo, con la perspectiva de ser rubricado en la cumbre de la Asociación Oriental de la UE de Vilnius en noviembre de 2013.

Por su parte, la Federación Rusa, que en 2010 lanzó la Unión Aduanera Rusia Belarusi, Kazajstán, pretendiendo constituir un contrapeso a la UE para los países vecinos, no ha dejado de intentar convencer a los demás estados que forman parte de la CEI, a unirse a la misma, utilizando en este dialogo incluso elementos de "hard power". El acercamiento de los países de la exURSS a Europa es percibido por Rusia como una amenaza de ver reducida o anulada su influencia en la región, por tanto hace esfuerzos para impedir a los países de la Asociación Oriental dar pasos en la dirección europea, y a la vez persuadirles a ser parte de la Unión aduanera.

Después de las polémicas decisiones en junio de los dirigentes de la autoproclamada república transnistrense de mover algunos puntos aduaneros hacia territorio moldavo, el

\footnotetext{
${ }^{24}$ Popescu, Nicu yWilson Andrew (2009): The limits of Enlargement-lite: European and Russian Power in the Troubled Neighbourhood, London, ECFR.

25 "Moldova elects president after 917-day deadlock", Euractiv.com, 19 March 2012, en http://www.euractiv.com/europes-east/moldova-elects-president-917-day-news-511565.

${ }^{26}$ Joint Statement by EU High Representative Catherine Ashton and Commissioner Štefan Füle on the noconfidence vote taken by the Moldovan Parliament on 5 March 2013, en http://www.consilium.europa.eu/uedocs/cms_Data/docs/pressdata/EN/foraff/135826.pdf.

${ }^{27}$ Discurso del Primer Ministro de Moldova, Iu. Leanca, designado en el Parlamento en la presentación del programa de gobierno y el nuevo gabinete de ministros, el 31 de mayo del 2013, en http://www.gov.md/libview.php?l=ro\&idc=436\&id=6403
} 
viceprimer ministro de Rusia emprendió una visita a Moldova y a la región de Transnistria. Su discurso ha contenido varias referencias críticas a la orientación europea de Moldova, así como expresiones que se han entendido y calificado de amenazas veladas del corte del suministro de gas de Rusia. ${ }^{28}$ La visita en los días siguientes del patriarca de la iglesia ortodoxa rusa a Moldova ha sido vista por muchos analistas como una herramienta del poder "blando" que Rusia sigue ejerciendo en su vecindad.

Pocos días más tarde Rusia anunció la interdicción de las importaciones de productos vinícolas moldavos en los mercados rusos, motivando que determinados procesos tecnológicos de producción no habían sido respetados. La región transnistrense, no obstante, quedaba excluida de la aplicación de este embargo.

A estas acciones se han sumado en las mismas fechas el comienzo de deportaciones de ciudadanos moldavos que están trabajando en del mercado laboral ruso, por razones de varios incumplimientos de las previsiones del régimen migratorio ruso. Aunque el presidente de la diáspora moldava en Rusia ha opinado que estos son los motivos reales de las medidas tomadas por la parte rusa, y no un instrumento más de presión sobre Moldova, la coincidencia en el tiempo de las medidas "disciplinarias" en diferentes áreas es difícil de obviar.

La posición de la Unión Europea con respecto a la actuación reciente de Rusia ha sido manifestada en una ponencia en el Parlamento Europeo del Comisario de Ampliación y Vecindad, Stefan Füle. El Comisario ha subrayado que cualquier forma de presión por parte de Rusia relacionada con la posible firma de acuerdos con la UE es inaceptable ${ }^{29}$. Por su parte, el Primer Ministro moldavo calificó el embargo de productos moldavos en el mercado ruso como "una decisión lamentable e infundada". 30

No obstante, a pesar de crisis políticas internas y de presiones externas, en la cumbre UE - Asociación Oriental, celebrada en Vilnius el pasado mes de noviembre, Moldova ha marcado un hito importante, rubricando el Acuerdo de Asociación con la UE.

\subsection{Dependencia energética: el gas como instrumento de influencia rusa}

Rusia intenta mantener continuamente la influencia en los países exsoviéticos y lo realiza por diferentes vías. Uno de sus instrumentos más eficientes hasta ahora ha sido el tema del abastecimiento energético. Es todavía reciente el recuerdo del conflicto del gas de enero de 2009 cuando por causa de problemas de impago y falta de suministro con Ucrania, Rusia recurrió a un drástico corte de importantes volúmenes de gas que se suministraban a través de Ucrania, y como resultado en 16 países europeos el abastecimiento energético se vio gravemente afectado en la temporada fría ${ }^{31}$.

\footnotetext{
${ }^{28}$ El alto cargo ruso bromeó diciendo "la energía es importante, sobre todo en vísperas del invierno, espero que no pasen Uds. frío", una alusión a la fuerte dependencia energética que experimenta Moldova, http://adevarul.ro/moldova/politica/dmitri-rogozin-1_5224cabbc7b855ff563b6c8f/index.html.

29 "Statement on the pressure exercised by Russia on countries of the Eastern Partnership", Stefen Füle, 11 September 2013, http://europa.eu/rapid/press-release SPEECH-13-687_en.htm

30 "El Primer ministro Leanca: El embargo impuesto a los vinos moldavos es una decisión lamentable e infundada", agencia de noticias UNIMEDIA, 11 de septiembre del 2013, en http://unimedia.info/stiri/premierulleanca-embargoul-impus-vinurilor-moldovenesti-este-o-decizie-regretabila-si-neintemeiata-65447.html.

31 SEC (2009) 977 final, "The January 2009 gas supply disruption to the EU: An assessment" en http://ec.europa.eu/energy/strategies/2009/doc/sec 2009 0977.pdf; "La guerra del gas entre Rusia y Ucrania provoca escasez en 16 países europeos", El País, 7 de enero de 2009, en

http://elpais.com/diario/2009/01/07/internacional/1231282806_850215.html.
} 
En cuanto a Moldova, la utilización del suministro energético como instrumento disciplinario en situaciones de divergencia ha sido frecuente. Uno de los casos recientes está relacionado con el contrato mediante el cual Chisinau importaba gas natural de Rusia que venció en enero de 2012, y desde entonces el suministro se hace en base a prorrogas contractuales de seis meses. La República de Moldova quiere un nuevo contrato para un máximo de cinco años y una reducción del precio del gas. En estos momentos toda Europa pasa por importantes cambios del mercado energético, las vías alternativas de suministro a Rusia están en construcción, y además una firme política de liberalización promovida por la UE contribuyen a la creación de un mercado energético común.

Hasta hace poco la empresa Gazprom tenía contrato con cada Estado Miembro por separado, pero a la vez con el mercado común la situación cambia y la interconexión se facilitarán las transacciones transfronterizas de la energía. En este contexto Rusia está descontenta con la decisión de la RM de firmar un contrato para solo cinco años dejándola así sin un importante instrumento de influencia y presión sobre este país. Para seguir manteniendo este instrumento de presión y hasta abuso en la región Rusia fue la que solicitó a la República de Moldova que renunciase a su propósito de adherirse al tercer paquete energético europeo, prometiéndole en cambio la concesión de un mejor precio para el gas suministrado, sacando también el tema de deuda transnistrense (que correría en opinión de Rusia a cargo de la RM, aunque en realidad Gazprom suministra la energía a la región, la población la paga y la administración separatista guarda el dinero para su presupuesto y la deuda acumulada la ha de pagar Moldova 3, 8 mil millones de dolares). El paquete energético III es la herramienta utilizada por la UE para liberalizar su mercado energético y luchar contra el poder de los monopolios en la región. La política energética es un área donde la República de Moldova ha hecho importantes cambios, llegando a ser miembro de la Comunidad energética europea, y por consiguiente está en camino de integrarse plenamente en las estructuras europeas desde el punto de vista energético. Este tipo de presiones por parte de Rusia, orientadas a impedir la integración energética de Moldova, levantan legítimas preocupaciones al respecto en cuanto pueden convertirse en fuertes instrumentos de influencia ${ }^{32}$.

\subsection{El conflicto transnistrense}

En septiembre de 1990 la administración de la región de Moldavia situada en la orilla izquierda del rio Nistru, con un gran número de rusoparlantes decidió no reconocer la legitimidad de los resultados de la elecciones parlamentarias de la República de Moldova en las cuales la mayoría fue obtenida por fuerzas políticas que optaban por la independencia y por una eventual re-unión con Rumanía ${ }^{33}$, una retórica frecuente en aquel período pero con poco fundamento real.

Después de dos años con un estatuto incierto, la región declaró su independencia de Moldova en 1992 y en aquel mismo período Rusia empezó a transportar a la región personal militar y armamento del Destacamento 14 del Ejército Ruso. Ulteriormente se produjeron choques entre las fuerzas paramilitares moldavas y las transnistrense y en marzo de 1992 estalló un conflicto armado que se llevó la vida de varios centenares de personas. El conflicto llevó a la dimisión del gobierno moldavo varios meses más tarde pero contribuyó a consolidar el régimen autoproclamado.

32 "Russia urges Moldova to renounce EU energy deal", Reuters.com, 12 September 2012, en http://www.reuters.com/article/2012/09/12/russia-moldova-gas-idUSL5E8KCC3F20120912.

${ }^{33}$ Steven, Roper D.: "Post-soviet Moldova's Identity and Policy" in Schmidtke, Oliver et. al., op.cit., pp.79-94. 
Con el apoyo de Rusia este estado de cosas sigue sin cambiar, a pesar de que ha habido diferentes etapas - desde períodos de tranquilidad hasta momentos de crisis en la evolución de las relaciones entre Moldova y la región transnistrense. Actualmente el conflicto congelado sigue siendo un serio obstáculo para las perspectivas del país de acercarse a la UE, constituye una importante fuente de inseguridad en la región, y por otra parte cumple el papel de factor de influencia de Rusia en la zona.

Durante casi todo este tiempo se ha considerado generalmente que es uno de los conflictos que podían ser resueltos sin mayores problemas, y con algunas interrupciones, las negociaciones entre Moldova y Transnistria, con la mediación de terceras partes (OSCE, UE, Ucrania, EEUU y Rusia) han continuado pero sin llevar al resultado esperado.

Los intereses de las partes son muy diferentes, y a la voluntad de Moldova de reintegración del país se opone la insistencia de la región de continuar siendo independiente, así como el interés de Rusia de que Transnistria como mínimo tenga un estatuto especial aunque sea en la composición de Moldova, y la Unión Europea y Ucrania, como vecinos directos de la región abogan por la resolución del conflicto mediante la reintegración de Moldova y por la liquidación por esta vía del foco de inseguridad.

En los últimos años se han dado nuevas condiciones y factores: la participación de la UE en el proceso de mantenimiento de la paz mediante la misión EUBAM ${ }^{34}$, la llegada al poder en Transnistria de representantes de una nueva generación con visiones más moderadas que el anterior poder, al igual que la ausencia de la componente de lucha étnica y la percepción que la población de las dos partes tienen sobre el problema (los ciudadanos moldavos sitúan el problema transnistrense en el noveno lugar de diez) ${ }^{35}$.

Los expertos opinan que la resolución del conflicto tiene mayores probabilidades desde un enfoque bottom-up, en el que apoyadas por la UE, Moldova y Transnistria lleguen a la reintegración mediante proyectos económicos comunes, mediante la consolidación de la confianza recíproca y mediante una mayor presencia de la UE en la región transnistrense ${ }^{36}$.

Mientras tanto, el problema sigue vigente, y aunque han pasado casi 20 años, las conversaciones entre las partes implicadas en el formato 5+2 no han podido llevar a las partes a un acuerdo sobre el estatuto del territorio. La región separatista sigue siendo una entidad no reconocida, autoproclamada y en cuyo territorio hay un vacío legal casi completo. Rusia

\footnotetext{
${ }^{34} \mathrm{CE}$, European Union External Action Service, http://www.consilium.europa.eu/eeas/security-defence/euoperations/moldova-and-ukraine-border-mission?lang=en: "The EU Border Assistance Mission to the Republic of Moldova and Ukraine was established in response to the joint letter from the Presidents of Moldova and Ukraine dating from 2 June 2005 calling for additional EU support for capacity building for border management, including customs, on the whole Moldova-Ukraine border, including the border between Ukraine and the separatist Transnistrian region of the Republic of Moldova, because the Moldovan border authorities are unable to be present there. On 7 October 2005, a Memorandum of Understanding on the Border Assistance Mission was signed between the European Commission and the governments of Moldova and Ukraine. The official opening ceremony of the EU Border Assistance Mission to Moldova and Ukraine took place on 30 November 2005. The mission is technical and advisory. Its mandate is to help improve the capacity of the Moldovan and Ukrainian border and customs services to prevent and detect smuggling, trafficking of goods and human beings, and customs fraud, by providing advice and training. The mission works very closely with the team of the EU Special Representative for Moldova Kálmán Mizsei, which has advisors in Kyiv, Chisinau and Odessa on political issues related to the border. The mission's mandate was extended twice - in 2007 and 2009. According to the latest agreement between the EU and the Governments of the Republic of Moldova and Ukraine the mission was prolonged until 30 November 2011".

${ }^{35}$ Popescu, Nicu y Litra, Leonid: "Transnistria: a bottom-up solution”, ECSF Report (September 2012), p.3.

${ }^{36}$ Ibid., p. 2.
} 
prefiere mantener el statu quo, porque puede utilizar la situación como una palanca de influencia en la zona cuando así lo requieren sus intereses, mientras que la UE que participa en el proceso e intenta ofrecer medidas para la resolución del conflicto, de momento parece no contar con una estrategia para este fin.

Mientras tanto Transnistria sigue siendo utilizada como instrumento de presión hacia Moldova por parte de Rusia y en estos casos la situación alcanza elevados niveles de tensión.

El pasado mes de junio la administración de la región transnistrense autoproclamada firmó un decreto de cambio de algunos puntos aduaneros en la frontera con Moldova, extendiéndose hacia algunas localidades de la jurisdicción de ésta, lo que provocó la preocupación de las autoridades que volvieron a insistir en la resolución pacífica de la situación $^{37}$ y la sociedad sobre la eventualidad de nuevos conflictos militares determinados por esta actuación unilateral.

La Federación Rusa, que apoya el régimen autoproclamado y mantiene en la zona fuerzas militares llamó a las partes a abstenerse de tomar acciones unilaterales que podrían llevar a un conflicto. Las autoridades moldavas calificaron las acciones de la región autoproclamada de un intento de desestabilizar el país con la ayuda de altos representantes de terceros estados, en clara alusión a Rusia.

\section{Situación de Ucrania}

La política de hacer equilibrio entre la Unión Europea y Rusia es un punto común de varios países de la Europa del Este, en particular de Ucrania y Moldavia. Los investigadores identifican tres razones por las que se persigue e intenta el mencionado equilibrio: primero la preferencia de las élites políticas que a los cambios y transformaciones prefieren el statu quo, por temor a que cualquier modificación les pudiera afectar sus negocios y posiciones; la segunda razón es de índole estructural: aunque la firma del Acuerdo de Asociación por parte de Ucrania por ejemplo, acerca a esta última a las instituciones y estructuras económicas de la UE. Este mayor acercamiento no siempre puede aparecer como una ventaja en el caso de Ucrania, país que tiene relaciones económicas de índole independiente con la UE, pero también en gran medida con Rusia y por tanto el país siempre intentará evitar que con un potencial acercamiento a una parte pueda perjudicar la buena relación con la otra y prefieren mantenerse en el papel de un país puente, ${ }^{38}$ la tercera razón que citan los autores es de orden contextual. En la actual dinámica de la economía global las élites de negocios del país ya no pueden esperar buenas noticias de parte de una Europa enfrascada en sus problemas de mercado sobre el acceso a mercados y créditos occidentales, lo que les determina a orientarse en busca de estas facilidades para su negocios en otra parte, y aquí interviene el mercado y los bancos rusos que están dispuestos a ofrecer los recursos y oportunidades necesarias a los emprendedores ucranianos.

Además de estas tres razones aquí podríamos añadir otra: el análisis coste-beneficio que hace Ucrania a la hora de llevar a cabo las transformaciones orientadas a acercarla a la Unión Europea, por ejemplo mediante la firma del DCFTA (Deep and Comprehensive Free Trade

\footnotetext{
${ }^{37}$ Declaración del Parlamento de la República de Moldova con respecto a la situación actual del proceso de reglamentación del conflicto transnistrense, Decisión del Parlamento 151 del 21 de junio del 2013, en http://lex.justice.md/index.php?action=view\&view=doc\&lang=1\&id=348411.

${ }^{38}$ Gnedina et al., op.cit, p. 2.
} 
Area) no son plenamente a favor de este acercamiento si consideramos sobre todo la dificultad que estos cambios supondrían a corto plazo para la economía ucraniana y la gran interdependencia económica que el país tiene con Rusia.

Dado que Rusia no está en absoluto a favor de la integración de Ucrania en la UE, esto podría llevarla a tomar medidas de presión que Ucrania difícilmente en estos momentos podría asumir - aumento del precio del gas, etc.

En cuanto a Ucrania, hasta hace dos años era el país sobre el que el Comisario S. Füle había comentado que la Unión Europea y Ucrania eran de enorme importancia la una para la otra $^{39}$, pero la situación cambió drásticamente en los últimos dos años.

Actualmente Ucrania, de sobra convencida de su importancia en la región y de su ubicación geográfica estratégica, intenta mantenerse en una independencia relativa tanto de la UE como de Rusia, prefiriendo acercarse a estos en los momentos cuando considera que el acercamiento le podría favorecer. En estos momentos el documento sobre la zona de comercio libre y comprehensivo está paralizado en una etapa próxima a la firma, los compromisos asumidos en los Planes de acción se han quedado realizados solo parcialmente. En vista de la posible adhesión de Ucrania a la Unión Aduanera organizada por Rusia, muchos activistas cívicos ucranianos veían como única solución para evitarlo la rápida firma del Acuerdo de Asociación con la UE. Aunque las negociaciones para dicho Acuerdo están bastante avanzadas, hay varios asuntos importantes que la UE no parece dispuesta a pasar por alto a la hora de acercar posiciones con Ucrania. La UE ha manifestado con antelación su preocupación y rechazo a la llamada "justicia selectiva" aplicada a la exprimer ministra ucrania Yulia Timoshenko ${ }^{40}$ y con respecto a las posibles irregularidades en el recuento de los votos en las últimas elecciones parlamentarias en este país. ${ }^{41}$ Después de la cumbre UE Ucrania del pasado mes de febrero, las preocupaciones siguieron siendo las mismas, y había pocas señales de la voluntad de Ucrania de actuar para cumplir las recomendaciones de la $\mathrm{UE}^{42}$

En los últimos meses Ucrania se ha visto en situaciones de presión por parte de Rusia, que intentaba por estos medios de persuadir a la administración del mayor país y el más avanzado de momento en la Asociación Oriental (tenía rubricado el Acuerdo de Asociación y sólo se esperaba que en la Cumbre de Vilnius se pudiera firmar) de que renunciara a la opción europea a favor de la adhesión a la Unión aduanera Rusia- Belarusi- Kazajstan.

Después de que reuniones ruso-ucranianas a alto nivel orientadas a convencer a Ucrania a adherir a la UA no se coronaron de éxito para Rusia, una de las primeras medidas fue la prohibición de la importación desde Ucrania de productos alimenticios (producción de una importante fábrica de bombones), más tarde a principios de agosto en la frontera de acceso en territorio ruso se han intensificado las verificaciones de productos y mercancías provenientes

\footnotetext{
${ }^{39}$ Füle, Stefan: “Address to the 14th European Union - Ukraine Parliamentary Cooperation Committee", Speech 10/114, Brussels, 22 March 2010.

${ }^{40}$ Comunicado de prensa del Parlamento Europeo en http://www.europarl.europa.eu/news/en/pressroom/content/20120523IPR45694/html/Parliament-demands-anend-to-selective-justice-in-Ukraine.

41 Ver más en Euractiv.com: http://www.euractiv.com/europes-east/eu-voices-concern-ukraine-electi-news$\underline{515826}$.

$\frac{515826}{42}$ "S. Füle's Speech in the European Parliament", 13 March 2013, en

http://europa.eu/rapid/press-release_SPEECH-13-226 en.htm
} 
de Ucrania. ${ }^{43}$ Estas acciones parecían haber tenido un resultado opuesto al esperado, ya que a principios del mes de septiembre los diputados de varias fuerzas políticas se habían consolidado para seguir votando leyes orientadas a la convergencia del marco legislativo ucraniano al europeo, de esta forma siguiendo su objetivo prioritario de acercarse a la UE.

Para la cumbre UE-Asociación Oriental (la iniciativa europea orientada a los países del Este de la PEV) celebrada el 28-29 de noviembre en Vilnius se planificaba que Ucrania iba a firmar el Acuerdo de Asociación. En las semanas anteriores se hablaba de la posible firma de documentos que supusieran la puesta en libertad de Yulia Timoshenko, ex primera ministra del país, cuyo caso es uno de los ejemplos de la "justicia selectiva" y en el que la UE insistía que se resolviera ${ }^{44}$. Pero la situación volvió a cambiar bruscamente una semana antes de la Cumbre, cuando la administración del país dio marcha atrás, decidiendo "suspender el proceso de preparación de la firma del AA" $" 45$. La principal razón que el poder de Ucrania indica es que es una decisión táctica, y que necesita revisar cómo las previsiones del Acuerdo de Asociación podrían influir en las condiciones de sus acuerdos con la Comunidad de Estados Independientes. Esto deja claramente al país en la zona de influencia de Rusia, cuyas presiones, junto con la situación interna de la clase dirigente del país han determinado este nuevo giro de Ucrania. Los analistas señalan la casi-previsible actuación-“escaqueo"46 a la que ha recurrido la administración de este país en diferentes momentos de su historia reciente.

La UE ha dejado claro su malestar con esta decisión de la administración de Ucrania ${ }^{47}$. Mientras tanto la desilusión del pueblo ucraniano con la actuación de sus dirigentes le ha hecho tomar las calles de Kiev en masivas protestas pacíficas, orientadas a manifestar la voluntad de la sociedad de seguir en la dirección de la UE, pero a los pocos días ya ha habido múltiples casos de intervención violenta de la policía contra los manifestantes.

\section{Aproximaciones combinadas: las ideas y los intereses}

En las relaciones de Moldova y Ucrania con la Unión Europea las dos partes han tenido comportamientos que se pueden explicar desde un enfoque racionalista y otros desde el enfoque constructivista, y que se han alternado en diferentes momentos del desarrollo de la relación.

Las teorías racionalistas alegan que las decisiones de formar parte de una organización internacional o supranacional se toman tanto por el estado que aspira a ser parte de la organización como por la organización respectiva después de hacer un análisis de la relación coste-beneficio que les compensa, y por tanto es una decisión que se toma a base de criterios meramente racionales. Este enfoque explica las actuaciones sociales mediante el interés por conseguir un objetivo individual. Los objetivos que se persiguen no son exclusivamente de

\footnotetext{
43 "Russia tightens customs rules to force Ukraine into union", Reuters, 15 August, 2013, en http://www.reuters.com/article/2013/08/15/russia-ukraine-customs-idUSL6N0GG17S20130815

${ }_{44}$ No release of Ukraine former PM, despite EU optimism, EUobserver.com, 16 October, 2013, en http://euobserver.com/foreign/121802.

45 "Cabinet Decides To Suspend Preparations For Signing Of Association Agreement With EU", Ukranian News Agency, 21 November 2013, en http://un.ua/eng/article/477766.html.

${ }^{46}$ Gnedina et. al., op.cit.

47 "Statement by EU High Representative Catherine Ashton on Ukraine", 21 November 2013, en http://eeas.europa.eu/statements/docs/2013/131121_04_en.pdf.
} 
orden material ni tampoco en interés propio - puede haber entre ellos objetivos normativos o de ideas, y se puede actuar en interés de otros. ${ }^{48}$

A diferencia del racionalismo, el constructivismo sostiene que las realidad social está construida por los actores parte de la sociedad mediante las prácticas cotidianas basadas en la importancia de las ideas compartidas e identidades. El enfoque constructivista se basa en la identificación de normas y valores compartidos para la actuación y la toma de decisiones de los actores sociales, y sin negar las realidades materiales destaca el factor humano y de creación de las ideas en la vida social ${ }^{49}$.

Kratochvil y Tulmets $^{50}$ y Schimmelfennig ${ }^{51}$ han analizado la actuación de las partes implicadas en el proceso: Unión Europea y estados no miembros.

Habitualmente al realizar un análisis muchos de los investigadores se orientan hacia una teoría u otra, aplicándola al caso que estudian.

En un principio se puede analizar la actuación de la UE hacia los países vecinos desde la perspectiva racionalista, ya que en virtud de las circunstancias históricas y políticas estos países vecinos no eran más que fuentes de inseguridad y conflictos en la frontera de los países europeo, y por consiguiente ofrecerles ayuda y asistencia respondía al interés de la UE. La condicionalidad, principio clave en la Política Europea de Vecindad y que supone mayor apoyo financiero y mayor colaboración para los países que cumplan mejor y de modo más eficiente las previsiones de los Planes de Acción es una instrumento basado en el principio racionalista. Los Planes Nacionales de Acción son documentos orientados a promover transformaciones políticas y democráticas, así como en una serie de políticas públicas de los países miembros de la PEV. Se puede ver la clara asimetría en los compromisos asumidos, correspondiéndole la mayor parte al país respectivo, cosa que aunque es lógica, dada la diferencia de poder de los actores, confirma en este contexto la actuación racionalista de la UE.

No obstante, el lanzamiento mismo de la Política Europea de Vecindad en los términos correspondientes puede ser visto desde un enfoque constructivista, como una acción determinada por la convicción de que aunque los países respectivos sean todavía muy diferentes y hayan formado parte de una entidad política totalitaria, el deber y los valores de la UE le determinan a hacer un paso de aproximación hacia estos países que necesitaban apoyo.

Por otra parte la actuación de los países vecinos, de su población pero también de sus élites políticas puede ser interpretada tanto desde el enfoque racionalista como desde el enfoque constructivista en diferentes etapas y circunstancias.

A la hora de manifestar claramente su voluntad de acercarse a Europa, a la UE, poco después de declarar su independencia, estos países compartían valores europeos y se identificaban a sí mismos como europeos. Su idea y convicción es que ellos siempre han formado parte de la cultura europea y por tanto su única vía para sobrevivir como nación era

\footnotetext{
48 Snidal, Duncan, "Rational choice and International Relations" in Carlsnaes, Walter; Risse, Thomas and Simmons, Beth, (eds.) (2008): Handbook of International Relations, Sage Publications, p. 75.

${ }^{49}$ Mariscal, Nicolás: “Aproximaciones constructivistas a la Unión Europea” en Cuadernos Europeos de Deusto, nr. 47, 2012, p.18.

${ }^{50}$ Kratochvil, Petr y Tulmets, Elsa, op. cit.

51 Schimmelfennig, Frank (2003): The EU, NATO and the Integration of Europe. Rules and Rhetoric, Cambridge, UK ; New York, Cambridge University Press.
} 
estar con Europa. La actuación y el discurso en aquel período y también más tarde estaban basados en convicciones, ideas y proximidades identitarias.

A la vez, la aceptación del principio de condicionalidad en los Acuerdos con la UE, que también puede ser vista como condicionalidad recíproca (no sólo el país recibe incentivos cuando hace reformas, sino también visto de otro modo - el país únicamente hace reformas si se le prometen incentivos) permite interpretar la actuación en los últimos años de los países respectivos, sobre todo de sus élites políticas, desde la perspectiva racionalista.

\section{2011 - año de crisis en la UE y en los países vecinos}

La crisis financiera y política en Europa ha afectado considerablemente a los países vecinos de la UE, poniendo de manifiesto una serie de puntos débiles de la Política Europea de Vecindad.

El hecho de tener que concentrarse en sus problemas internos ha determinado una mayor reserva por parte de la UE a la hora de aumentar el volumen de la asistencia financiera a los países vecinos.

Otro problema que ha salido a la superficie a raíz de la crisis económica es la falta de convergencia entre los países miembros a la hora de coordinar la asignación de la financiación para estos instrumentos, lo que tradicionalmente llevaría a la puesta en tela de juicio de las futuras perspectivas de ampliación.

Pero donde mayor impacto negativo ha tenido la crisis, y así lo señalan varios autores ${ }^{52}$, es en el debilitamiento de la condicionalidad como principal instrumento de la política que unos llaman más exitosa de la UE - la política de ampliación. Esto reduce considerablemente el atractivo de la UE en la región, sobre todo en el contexto de un mundo multipolar. Refiriéndonos a los países que nos conciernen, un importante polo de atracción en las condiciones de pasividad de la UE es Rusia. Como país heredero del imperio soviético, Rusia se esfuerza por mantener su presencia y sobre todo influencia en la zona, utilizando la táctica de los incentivos y las presiones - el palo y la zanahoria. En cuanto a los incentivos, las fuentes citadas anteriormente mencionan que Rusia tiende a ofrecer mayores incentivos que la Unión Europea, adquiriendo así mayor influencia y cobrando atractivo y adeptos tanto entre la población como entre algunos representantes de la élite política. Popescu y Wilson señalan que la UE tiende a confiar demasiado a veces en la importancia de su presencia en algunos de estos países y también en la fuerza del convencimiento, de los valores y del llamado poder blando. Los autores recomiendan que a la luz de la importancia que tiene para la Unión la existencia en sus fronteras de países democráticos y seguros, se intentaran emprender acciones orientadas a consolidar no sólo la presencia sino también el poder, incluso mediante la concesión de incentivos esperados por los países respectivos.

Otro factor importante a la hora de explicar el papel menos influyente de la UE en los países vecinos es el contexto multipolar que se crea últimamente: EEUU que aunque esporádicamente, casi siempre han seguido teniendo influencia y en cuanto a nuevos polos de poder - China es uno de ellos, que concede ayuda al régimen de Lukashenko en Belarus. En

\footnotetext{
${ }^{52}$ Whitman, Richard y Juncos, Ana: "The Arab Spring, the Eurozone Crisis and the Neighbourhood: A Region in Flux", in JCMS 2012, Volume 50 Annual Review p. 149; Popescu y Wilson: "Turning presence...", op.cit., p. 61.
} 
cuanto a Rusia, siempre ha estado presente, alternado el poder duro (la guerra en Georgia) con elementos de poder blando (facilidades en comercio o en áreas de derecho en los países de su vecindad cercana).

\section{Conclusiones}

\section{¿Seguirá siendo Moldova una historia de éxito?}

En las condiciones cuando Ucrania ya no parecía tan convencida de su orientación europea, salvo a nivel de discurso, Moldova había dado la impresión en los últimos años de avanzar con pasos firmes en la senda anunciada de acercamiento a la UE, asumiendo e implementando reformas, esforzándose por mejorar las estructuras y el nivel de eficiencia de las instituciones del estado, etc. La UE a su vez también ha destacado esta voluntad y esfuerzo de Moldova, como hemos indicado anteriormente, dándole claras señales de aprobación y apoyo. En opinión de algunos analistas, la UE necesitaba una historia de éxito ${ }^{53}$ en la PEV para confirmar la utilidad y adecuación de esta política, y Moldova parecía que se acercaba a serlo. La crisis política surgida el 5 de marzo de 2013 con la aprobación de la moción de censura y la dimisión del Gobierno de Moldova vuelve a confirmar la tesis anteriormente emitida.

No obstante los constantes altibajos políticos, la República de Moldova ha manifestado y mantenido su vocación europea desde la obtención de su independencia.

Mediante la Política Europea de Vecindad la Unión Europea ha intentado ofrecerles a los países de su vecindad un marco para la asistencia y ayuda en la promoción de la democracia, para las reformas institucionales y el desarrollo económico y social.

Para los países que han manifestado su interés de formar parte de la UE como son Moldova y Ucrania la Política ha sido un buen instrumento de evolución y desarrollo, pero les ha causado también frustraciones por no haberse cumplido las mayores expectativas que tenían. Por otra parte, la PEV es una política en desarrollo, y no es ni una política de ampliación ni política exterior ${ }^{54}$.

Aun siendo un país comprometido con la orientación europea y encaminado hacia las reformas requeridas para el acercamiento, Moldova no obstante, ha tenido y sigue teniendo una trayectoria zigzagueante en cuanto a la orientación de su política exterior se refiere. Hemos identificado en el artículo algunos de los motivos de esta actuación. Entre las razones internas podríamos señalar la relativamente corta historia y tradición democrática del país con las consiguientes débiles estructuras institucionales, alto nivel de corrupción en algunas de estas y la insuficiente preparación y la carencia de valores de gran parte de la clase política.

La influencia de Rusia en la región constituye una razón desde el exterior para la oscilación del vector de orientación moldavo y también ucraniano. Siguiendo su objetivo de mantener su influencia en todos los ámbitos de estos países - económico, cultural, social Rusia cuenta para esto con poderosos instrumentos de poder - la dependencia energética, la presencia militar en Transnistria y también instrumentos de convicción más blandos apertura del mercado laboral y la influencia cultural fruto de un pasado común.

\footnotetext{
${ }^{53}$ Boonstra Jos: "Moldova: a success story?", Policy brief, FRIDE (2011).

${ }^{54}$ Missiroli, Antonio: "The ENP in Future Perspective" en Whitman, Richard y Wolff, Stefan, (eds.) (2010): The ENP in perspective. Context, Implementation and Impact, Palgrave Macmillan, pp. 262-263.
} 
Aun así desde 2010 Moldova, motivada por las mayores perspectivas de acercamiento a la UE ("más por más" 55 ) ha realizado importantes reformas en sus estructuras de estado, en sus políticas públicas y ha consolidado su democracia, y mediante la puesta en marcha de las negociaciones para el Acuerdo de Asociación la UE le ha reconocido estos avances. El dialogo político entre la UE y Moldova ha continuado hasta 2013 y las negociaciones para el Acuerdo de Asociación avanzaron con éxito.

En vísperas de la Cumbre UE - Asociación Oriental la UE propuso el levantamiento de los visados para los ciudadanos moldavos con pasaportes biométricos, confirmando así los logros de la República de Moldova en el proceso de reformas internas asumidas mediante el Plan de Acciones para la liberalización de visados. ${ }^{56} \mathrm{Si}$ se consiguiera la aprobación de la propuesta, Moldova sería el único país de la ex URSS (además de las exrepúblicas bálticas) en obtener el derecho de viajar a la UE sin visados ${ }^{57}$, y además este sería un beneficio tangible del acercamiento a la Unión para sus ciudadanos. Dicha propuesta es una importante señal por parte de la UE de reconocimiento y apoyo de las reformas que Moldova está llevando a cabo.

Aunque actualmente el país está considerado el front runner de la Asociación Oriental $^{58}$, su posición sigue siendo frágil. La evolución favorable lograda hasta la fecha puede verse puesta en peligro por una nueva crisis política (bastante frecuentes en este tipo de contextos) que podría cambiar la orientación de Moldova en una dirección contraria a la UE.

Como hemos indicado, para Rusia el acercamiento de la Moldova hacia la Unión Europea equivale a la pérdida de la influencia en la zona y por consiguiente la determina a tomar medidas de diferente índole: desde la propuesta a unirse a la Unión Aduanera que traería beneficios económicos para Moldova y hasta las citadas prohibiciones de las importaciones y la instigación a tensiones en la región transnistrense, la cual sigue siendo una de las herramientas principales de influencia rusa.

Frente a las acciones de Rusia hacia Moldova y Ucrania en vísperas de la Cumbre de Vilnius, donde se esperaba marcar un importante hito en la trayectoria europea de estos dos países - la rúbrica y la firma respectivamente de unos Acuerdos de Asociación, la UE ha hecho declaraciones orientadas a apoyarlos.

En el citado comunicado del Comisario Stefan Füle el oficial europeo ha puesto de manifiesto que aunque la Unión Aduanera y los Acuerdos de Asociación no son compatibles de ser firmados por un país, se debe dejar a los países vecinos a ejercer libremente su derecho a tomar decisiones como estados soberanos que son, y a la vez crear, donde se puedan, nuevos puntos de colaboración y cooperación entre los países vecinos y Rusia, la cual a su vez también podrá beneficiarse de la integración de los países de la Asociación Oriental en la economía europea.

\footnotetext{
${ }^{55}$ Comunicación de la CE, JOIN (2012) 14 final, "Diseño de una nueva Política Europea de Vecindad"

56 "Commission proposes visa-free regime to Moldova", Press Release, 27 November 2013, en http://europa.eu/rapid/press-release_IP-13-1170_en.htm.

57 "The Surprising Front-Runner. Moldova Before and after Vilnius Summit", ESI Discussion Paper, 2 December 2013, en http://www.esiweb.org/pdf/esi_document_id 146.pdf.

58 "Moldova front-runner of Eastern Partnership, Van Rompuy tells Prime Minister Leanca", EU Neighbourhood Info, 18 November 2013, en

http://enpi-info.eu/maineast.php?id=35159\&id_type=1\&lang_id=450.
} 
Según un análisis realizado por el think tank moldavo "Expert Grup" 59 las dos opciones de integración que afronta Moldova - por una parte la Unión Aduanera Rusia- BielarusiKazajstan y por otra la firma del Acuerdo de Asociación con la UE - parecen ofrecer determinadas ventajas y desventajas al país. El estudio concluye no obstante que la firma del Acuerdo de Asociación e implícitamente del DCFTA (Deep and Comprehensive Free Trade Area) tendrá efectos mucho más positivos para la economía moldava, sólo que estos serían visibles / notables a largo plazo, mientras que a corto plazo algunos sectores de la agricultura, por ejemplo, podrían incluso sufrir pérdidas causadas por el aumento de la competencia.

Entre las consecuencias o efectos que las opciones tendrían para la política exterior de Moldova los autores señalan que la eventual adhesión de Moldova a la Unión Aduanera sería incompatible con la firma del Acuerdo de Asociación, implícitamente con el acercamiento a la UE que ha sido el objetivo prioritario en los últimos años, de esta forma se pondría en riesgo el proceso de reformas orientadas a la transformación de Moldova según el modelo europeo. Al mismo tiempo la firma del AA no supondría para Moldova la imposibilidad de mantener la cooperación económica con Rusia y con otros países miembros de la Comunidad de Estados Independientes.

Aun así, Rusia ve peligrada su posición de influencia en la zona y toma medidas para mantenerla a toda costa.

Los países de la Asociación Oriental más avanzados en el proceso de acercamiento a la UE depositaban grandes esperanzas en la Cumbre de Vilnius como un hito y un punto irreversible en su relación con la Unión, en la vía de europeización y la ansiada perspectiva de futura adhesión.

A pesar de los grandes avances en la relación de la UE con los países de la Asociación Oriental, varios analistas subrayan la dificultad de la situación. La región de la Asociación Oriental es propensa a crisis y a la inestabilidad interna, política, pero también geopolítica en virtud de su ubicación. Moldova ha sufrido en el último año una prolongada crisis política interna, mientras que Ucrania sigue teniendo un problema serio en el aspecto de la justicia selectiva y la situación de Timoshenko. Este tipo de problemas, unidos a la precaria seguridad energética y económica, junto con la influencia de Rusia en Transnistria pueden poner en peligro los avances realizados hasta la fecha. La presión ejercida por Rusia ha dado sus frutos en Armenia, uno de los socios de la Asociación Oriental con progresos más rápidos en el proceso de acercamiento a la UE, que tenía la perspectiva de rubricar el Acuerdo de Asociación en la cumbre de Vilnius, pero también un país con fuerte dependencia energética y militar de Rusia, ha declarado inesperadamente su adhesión a la Unión Aduanera. Como hemos visto, también Ucrania, pocos días antes de firmar el AA, ha cedido a sus presiones. En cuanto a Moldova, que se plantea firmar el Acuerdo dentro de un año, los analistas opinan que tampoco se va a ver exenta de dificultades y presiones tanto externas como internas en este período.

Dados los factores de inestabilidad y presión en democracias todavía inestables, como las que estamos analizando, se hace difícil prever evoluciones a medio y largo plazo.

\footnotetext{
${ }^{59}$ Lupusor, Adrian; Cenusa, Denis y Fala, Alexandru: “Quo vadis Moldova: European integration, euroasiatic integration or statu quo?”, Expert-group publications (September 2013), en

http://expert-grup.org/en/biblioteca/item/849-quo-vadis-moldova-integrarea-european\%C4\%83-integrareaeuroasiatic\%C4\%83-sau-status-quo/849-quo-vadis-moldova-integrarea-european\%C4\%83-integrareaeuroasiatic\%C4\%83-sau-status-quo?category=7.
} 
Por tanto el papel que le corresponde a la UE en el proceso de evolución de Moldova y Ucrania es difícil de sobrevaluar. El interés de Europa de mantener una buena relación política y económica con los países de la región, así que como el atractivo del modelo europeo y la motivación de cambio que este determina en estos estados pueden ser herramientas eficientes a la hora de ayudarlos a mantenerse en la órbita de la estabilidad y en la senda de desarrollo democrático, social y económico. 